\title{
Bursectomy and non-bursectomy D2 gastrectomy for advanced gastric cancer, initial experience from a single institution in China
}

\author{
Wei-Han Zhang ${ }^{1,2}$, Xin-Zu Chen ${ }^{1,2}$, Kun Yang ${ }^{1,2}$, Kai Liu ${ }^{1,2}$, Zhi-Xin Chen ${ }^{1}$, Bo Zhang ${ }^{1}$, Zong-Guang Zhou ${ }^{1}$
} and Jian-Kun $\mathrm{Hu}^{1,2^{*}}$

\begin{abstract}
Background: The aim of this study is to evaluate the safety and efficacy of bursectomy of D2 gastrectomy in terms of postoperative complications and short-term survival outcomes.

Methods: From January 2012 to December 2013, data of 406 gastric cancer patients with advanced tumor stages and who underwent D2 radical gastrectomy and were grouped according to whether bursectomy was performed or not in West China Hospital, Sichuan University, were analyzed.

Results: Finally, 159 patients were in bursectomy group and 247 patients in non-bursectomy group. Surgical duration was $260.1 \pm 43.4 \mathrm{~min}$ in the bursectomy group, compared to $227.9 \pm 48.6 \mathrm{~min}$ in the non-bursectomy group $(p<0.001)$. The intraoperative blood loss was comparable between the bursectomy group and the non-bursectomy group (198.9 \pm 63.5 vs. $201.1 \pm 53.7 \mathrm{ml}, p=0.729$ ). Postoperative morbidity rate showed no significant difference between the two groups, which were $23.3 \%$ in the bursectomy group and $17.8 \%$ in the non-bursectomy group, $p=0.179$. The overall survival outcomes of patients were compared between the two groups of all patients $(p=0.055)$ : patients who underwent distal gastrectomy $(p=0.129)$ and total gastrectomy $(p=0.016)$ and $p T 2-3$ stage patients $(p=0.117)$ and $\mathrm{pT} 4 \mathrm{a}$ stage patients $(p=0.128)$. The multivariate survival analysis identified that bursectomy or not, $\mathrm{pT}$ stage and $\mathrm{pN}$ stage were independent prognostic risk factors for the overall survival.
\end{abstract}

Conclusions: The bursectomy might increase the surgical duration when the D2 gastrectomy was done. Experienced surgeons can perform it safely. However, for the survival benefits of bursectomy, long-term, large sample sized, and high-quality randomized controlled trials are expected.

Keywords: Gastric cancer, Gastrectomy, Bursectomy, Complication, Prognosis

\section{Background}

Gastric cancer is the second most common cause of cancer-related death due to its malignant potential [1-3]. Owing to the absence of large sample size prospective randomized control trials with long-term survival results, there have been many disputes on the clinical use of bursectomy with gastrectomy for advanced gastric cancer

\footnotetext{
*Correspondence: hujiankun126@126.com

'Department of Gastrointestinal Surgery, West China Hospital, Sichuan University, Chengdu, Sichuan Province, China

2Laboratory of Gastric Cancer, State Key Laboratory of Biotherapy, West China Hospital, Sichuan University, Chengdu, Sichuan Province, China
}

patients. Bursectomy is mainly defined as a complete dissection of the peritoneal lining covering the pancreas and the anterior plane of the transverse mesocolon and with an omentectomy during gastrectomy $[4,5]$. This surgical technique has been developed as a part of radical gastrectomy with the aim of removing the potential microscopic tumor seeding since the 1960s in Japan and based on the following oncological and anatomical theories [6-8]: (1) prevents peritoneal recurrences by eliminating micrometastatic disease in the lesser sac of peritoneal cavity and (2) complete resection of the subpyloric lymph nodes (LNs). However, the therapeutic value of bursectomy is

\section{Biomed Central}

(c) 2015 Zhang et al. Open Access This article is distributed under the terms of the Creative Commons Attribution 4.0 International License (http://creativecommons.org/licenses/by/4.0/), which permits unrestricted use, distribution, and reproduction in any medium, provided you give appropriate credit to the original author(s) and the source, provide a link to the Creative Commons license, and indicate if changes were made. The Creative Commons Public Domain Dedication waiver (http://creativecommons.org/publicdomain/zero/1.0/) applies to the data made available in this article, unless otherwise stated. 
controversial because the survival benefit is uncertain. One randomized controlled trial performed by the Osaka University Clinical Research Group found that bursectomy may improve the survival outcomes in pT3-4 stages patients and should not be dropped [9]. However, other two retrospective studies indicated that there were no survival benefits for bursectomy when compared with nonbursectomy for gastric cancer patients [10, 11]. According to a recently meta-analysis that included one randomized controlled trial (RCT) and three non-RCTs, Shen et al. found that there was no survival benefits for the bursectomy when compared with non-bursectomy surgery for gastric cancer patients and the bursectomy was not recommended as a routinely procedure for gastric cancer surgery [12]. Meanwhile, the treatment guideline of Japanese Gastric Cancer Association (JGCA) only recommended that bursectomy can be selectively used according to the specific tumor stage and location $[13,14]$.

Due to the high proportion of advanced stage patients in China, we analyzed the clinicopathological data, the postoperative complications and survival outcomes for advanced gastric cancer patients who underwent D2 radical distal and total gastrectomy with or without bursectomy to report the initial experience of our center.

\section{Methods}

\section{Ethical statement}

The Ethics Committee of West China Hospital, Sichuan University, approved this retrospective study. The participants' written consent were not obtained, but the patients' records were anonymous to analysis.

\section{Patients}

From January 2012 to December 2013, those gastric cancer patients from the Department of Gastrointestinal Surgery, West China Hospital, Sichuan University, were included in this study based on the following criteria: (1) histologically confirmed gastric adenocarcinoma; (2) pT24, N0-3, and M0 stages according to the Japanese Classification of Gastric Carcinoma [15]; (3) distal gastrectomy and total gastrectomy by the conventional open method; (4) D2 lymphadenectomy according to the Japanese gastric cancer treatment guidelines [13]; and (5) curative resection without residual tumors (R0 resection). And those patients with distant metastases or positive cytology examination were excluded. Those patients underwent preoperative adjuvant chemotherapy were excluded to minimize the survival influence of it. Finally, the records of the patients who fulfilled the inclusion and exclusion criteria for this study were obtained and analyzed.

\section{Bursectomy and operative procedure}

All the gastric cancer patients included in this study had undergone surgical treatments by well-trained surgeons in the gastric cancer treatment team of our department. Because this is not a randomized controlled study, patients who underwent bursectomy or non-bursectomy surgery were intraoperatively decided by the chief surgeons. The surgical treatment principle was adopted by the Japanese Gastric Cancer Treatment Guidelines which was published by the JGCA [16]. For the resection patterns, those advanced gastric cancer patients with tumors located in the upper or middle third had total gastrectomy. Patients with tumors located in the lower third of the stomach and with lymph nodes metastasis in no. 1, no. 2 , and no. $4 \mathrm{sb}$ stations during the intraoperative frozen section evaluation also underwent total gastrectomy. Only lower third gastric cancers and distal gastrectomy can secure the tumor-free radical resection and have distal gastrectomy. According to the guidelines of the JGCA, in order to attain the complete dissection of subpyloric lymph nodes, the right side of the anterior plane of the transverse mesocolon and the pancreas was routinely resected, which was partially bursectomy. Besides the right side of the bursa omentalis, total bursectomy should be with en bloc resection of the peritoneal lining of the bursa omentalis (the anterior lobe of the transverse mesocolon and the pancreas), and the anterior lobe of the transverse mesocolon and the capsule of the pancreas should be dissected as much as possible [10], which was defined as the total bursectomy. Patients who underwent partial bursectomy (right side) were included in the non-bursectomy group, and patients who underwent total bursectomy were grouped in the bursectomy group. During the surgical procedures, the exposure of the surgical field, appropriate tension is essential to the success of completely removing the anterior lobe of the transverse mesocolon and the capsule of the pancreas (Fig. 1). Therefore, when the bursectomy is finished, only the posterior layer of the transverse mesocolon remained and the anterior lobe of the transverse mesocolon and the capsule of the pancreas were entirely separated from the transverse mesocolon and the pancreas (Fig. 2). Additional surgical procedures between the two groups were similar. D2 lymphadenectomy was carried out according to treatment guidelines published by Japanese Gastric Cancer Association [13]. Roux-en-Y esophagojejunostomy reconstructions were for total gastrectomy. And for distal gastrectomy, Billroth type I/II or Roux-en-Y gastrojejunostomy reconstructions were performed according to the tumor characteristics and others.

\section{Clinicopathological characteristics}

Clinicopathological characteristics, perioperative morbidity, and mortality were analyzed. The cross-sectional location and the longitudinal location of tumors were recorded according to the standard of the Japanese Classification of Gastric Carcinoma [15]. Lymph nodes were separately examined by the anatomical definitions of lymph node 


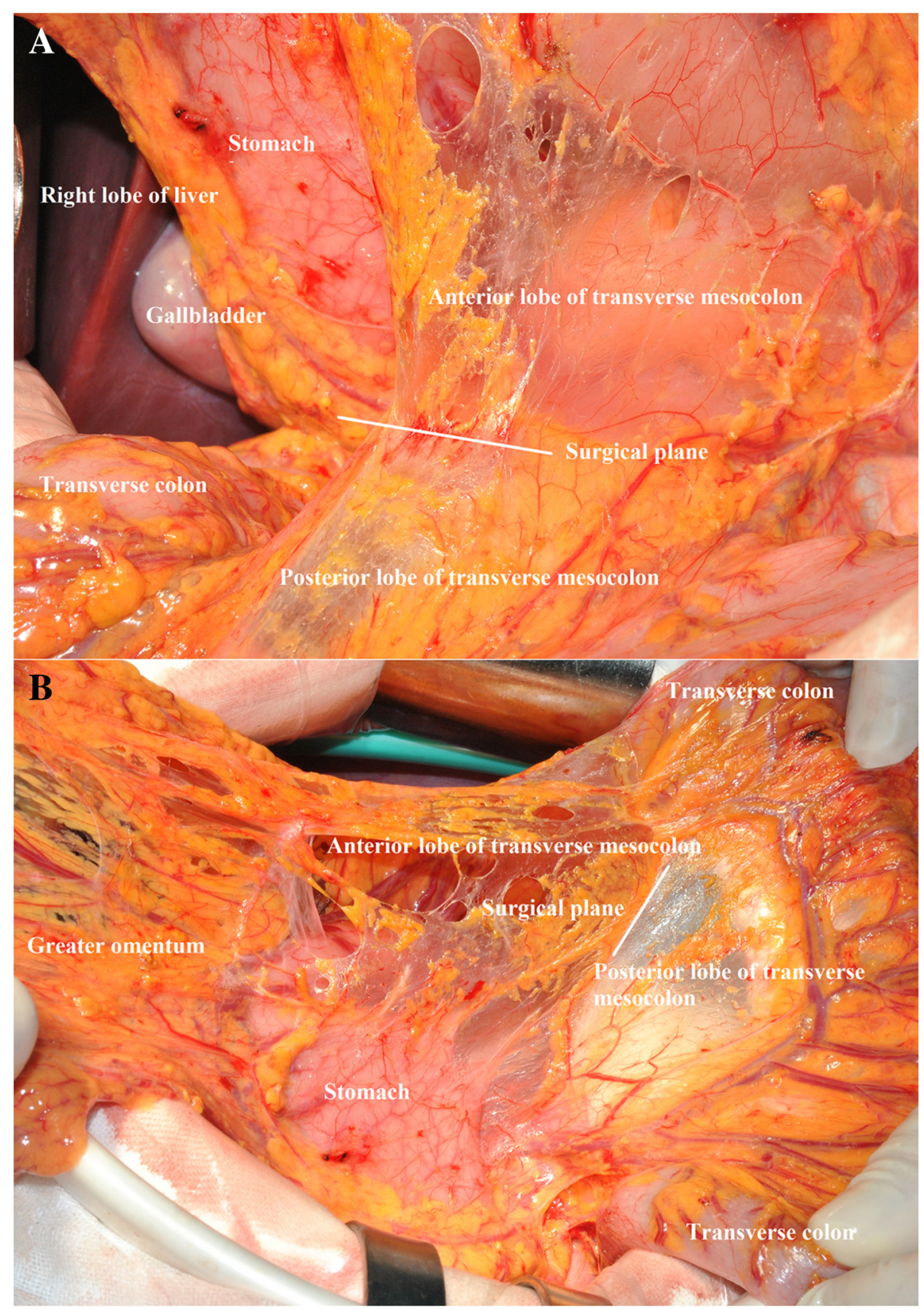

Fig. 1 Removal of the anterior lobe of the transverse mesocolon. a The surgical plane of the removal of the anterior lobe of the transverse mesocolon (front view). $\mathbf{b}$ The surgical plane of the removal of the anterior lobe of the transverse mesocolon (side view)

stations, according to the Japanese guidelines [13]. The number of positive and examined lymph nodes of the no. $4 \mathrm{~d}$, no. 4sb, and no. 6 LNs were compared between the two groups. The cancers were staged according to the Union for International Cancer Control (UICC) tumor-nodemetastasis (TNM) system, 7th Edition [17]. Postoperative mortality and morbidity were counted for 30 days or during the same hospitalization. Specifically, the classification of pancreatic fistula was according to the international study group of pancreatic fistula classification [18]. The grades of the postoperative complications by the ClavienDindo classification of surgical complications between the two groups were evaluated and compared [19].

\section{Follow-up}

The follow-up was performed by means of routinely outpatient visit. Mail and telephone interviews were the supplementary methods. The follow-up information was 


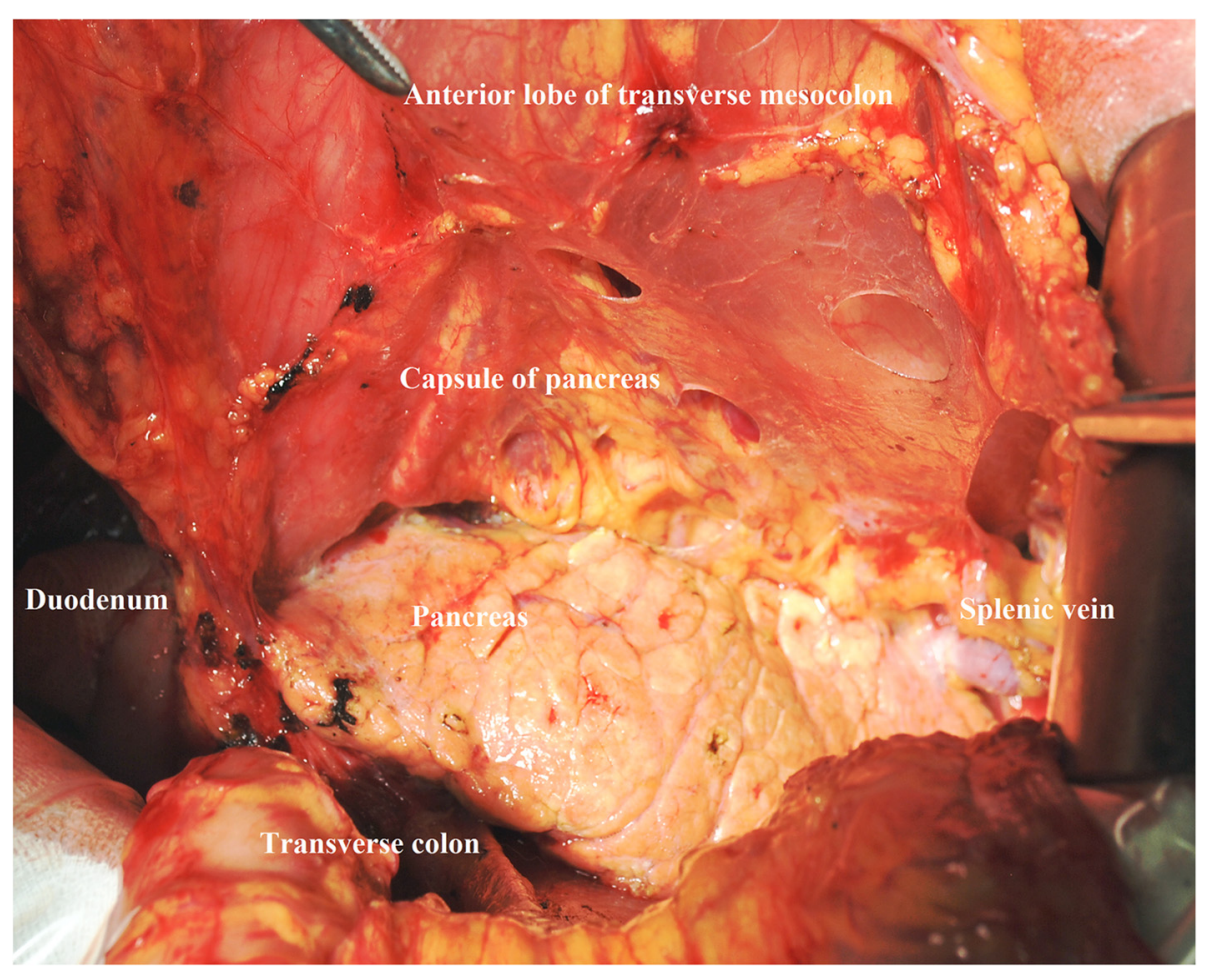

Fig. 2 Complete removal of the capsule of the pancreas and the anterior lobe of the transverse mesocolon

updated until January 1, 2015. The follow-up rate, median follow-up duration (months), recurrence type, and overall survival outcomes were analyzed in the study. Recurrence types include the local-regional recurrence (anastomotic recurrence) type, peritoneal seeding recurrence type, hematogenous recurrence (liver, pulmonary, bone, et al.) type, distal lymph nodes (non-regional lymph nodes) type, and multisite recurrence type. The reasons for the patients lost to follow-up were predominantly refusal of the outpatient visit or a change in the telephone number and address.

\section{Statistics}

Statistical analysis was conducted using SPSS statistics software, version 19.0 (SPSS, Chicago, Illinois, USA). For the continuous variables with normal distribution, they were analyzed by the one-way ANOVA test. Both the continuous variables without normal distribution and the ranked variables were weighed by the Mann-Whitney $U$ test. The categorical variables were adopted with the Pearson's chi-square test (or likelihood ratio). Survival outcomes were reported to Kaplan-Merrier method, log-rank test. Multivariate adjusted factor survival analysis was performed using Cox proportional hazard modeling. Hazard ratio (HR) and $95 \%$ confidential interval (CI) were used to present the results of the univariate and multivariate survival analysis. Two-tailed $p$ value of less than 0.05 was regarded as statistically significant.

\section{Results}

\section{Clinicopathological features}

From January 2012 to December 2013, 406 gastric cancer patients were included in the final analysis, 159 of whom in bursectomy group and 247 in non-bursectomy group. Most of the clinicopathological characteristics were comparable between the two groups, such as age, gender, tumor size, and tumor locations, and as well as tumor stages (Tables 1 and 2). Surgical duration was significantly increased in the bursectomy group when compared with the non-bursectomy group $(260.1 \pm 43.4$ vs. $227.9 \pm 48.6 \mathrm{~min}, p<0.001$, respectively). However, the amount of blood loss was comparable between bursectomy group than the non-bursectomy group $(198.9 \pm 43.4$ vs. $201.1 \pm 53.7 \mathrm{ml}, p=0.729$ ). Meanwhile, we found out that more proportion of patients underwent total gastrectomy in bursectomy group than non-bursectomy group (54.1 vs. $25.5 \%, p<0.001$ ). Also, there existed difference in the longitudinal location of tumors (upper, middle, lower) between the bursectomy and nonbursectomy groups $(39.0,13.8,47.2 \%$ vs. 11.2, 18.2, $70.4 \%$, respectively, $p<0.001)$.

\section{Lymph node status}

Total number of examined LNs was significantly higher in bursectomy group than non-bursectomy group (40.6 \pm 17.5 vs. $25.4 \pm 9.9, p<0.001)$. Besides, the number of positive lymph nodes was also higher in the bursectomy group 
Table 1 Clinicopathological characteristics of the nonBursectomy group and the bursectomy group

\begin{tabular}{|c|c|c|c|}
\hline \multirow[t]{2}{*}{ Characteristics } & \multirow{2}{*}{$\begin{array}{l}\text { Non-bursectomy } \\
\text { group } \\
N=247(\%)\end{array}$} & \multirow{2}{*}{$\begin{array}{l}\text { Bursectomy } \\
\text { group } \\
N=159(\%)\end{array}$} & \multirow{2}{*}{$p$ value } \\
\hline & & & \\
\hline Age (years) & $58.3 \pm 12.2$ & $57.3 \pm 11.5$ & 0.385 \\
\hline Gender & & & 0.513 \\
\hline Male & $160(64.8)$ & $108(67.9)$ & \\
\hline Female & $87(35.2$ & $51(32.1)$ & \\
\hline Cross-sectional location & & & 0.008 \\
\hline Lesser & $124(50.2)$ & $101(63.5)$ & \\
\hline Greater & $15(6.1)$ & $11(6.9)$ & \\
\hline Anterior & $34(13.8)$ & $8(5.0)$ & \\
\hline Posterior & $40(16.2)$ & $15(9.4)$ & \\
\hline $\begin{array}{l}\text { Circumferential } \\
\text { involvement }\end{array}$ & $34(13.8)$ & $24(15.1)$ & \\
\hline Longitudinal location & & & $<0.001$ \\
\hline$U$ & $28(11.2)$ & $62(39.0)$ & \\
\hline M & $45(18.2)$ & $22(13.8)$ & \\
\hline L & $174(70.4)$ & $75(47.2)$ & \\
\hline Differentiation grade & & & 0.328 \\
\hline Well-moderate & $29(11.7)$ & $24(15.1)$ & \\
\hline Poor-undifferentiated & $218(88.3)$ & $135(84.9)$ & \\
\hline Macroscopic type & & & 0.307 \\
\hline Type 1 & $14(5.7)$ & $4(2.5)$ & \\
\hline Type 2 & $99(40.1)$ & $75(47.2)$ & \\
\hline Type 3 & $118(47.8)$ & $71(44.7)$ & \\
\hline Type 4 & $16(6.5)$ & $9(5.7)$ & \\
\hline Tumor size (cm) & $5.7 \pm 2.4$ & $5.6 \pm 2.3$ & 0.537 \\
\hline Surgical duration (min) & $227.9 \pm 48.6$ & $260.1 \pm 43.4$ & $<0.001$ \\
\hline Blood loss (ml) & $201.1 \pm 53.7$ & $198.9 \pm 63.5$ & 0.729 \\
\hline Resection patterns & & & $<0.001$ \\
\hline DG & $184(74.5)$ & $73(45.9)$ & \\
\hline TG & $63(25.5)$ & $86(54.1)$ & \\
\hline
\end{tabular}

Abbreviations: $U$ upper, $M$ middle, $L$ lower, $D G$ distal gastrectomy, $T G$ total gastrectomy

than the non-bursectomy group $(7.5 \pm 8.7$ vs. $5.9 \pm 6.4$, $p=0.045)$. Comparison of the number of the positive LNs and the number of examined LNs between the two groups in no. $4 \mathrm{~d}$ LNs, no. $4 \mathrm{sb}$ LNs, and no. 6 LNs were listed in Table 2. The number of examined LNs was $4.9 \pm$ 4.0 in no. $4 \mathrm{~d}$ LNs of bursectomy group compared to $3.7 \pm$ 2.7 of non-bursectomy group, $p<0.001$. The number of examined LNs in no. 4SB LN and no. 6 LNs was similar between the two groups, $p=0.743$ and $p=0.362$, respectively.

\section{Mortality and morbidity}

There was no incidence of intraoperative mortality of patients in this study. The mean postoperative hospital stay
Table $\mathbf{2}$ Lymph node status of the non-bursectomy group and the bursectomy group

\begin{tabular}{|c|c|c|c|}
\hline \multirow[t]{2}{*}{ Characteristics } & $\begin{array}{l}\text { Non-bursectomy } \\
\text { group }\end{array}$ & $\begin{array}{l}\text { Bursectomy } \\
\text { group }\end{array}$ & \multirow[t]{2}{*}{$p$ value } \\
\hline & $N=247(\%)$ & $N=159(\%)$ & \\
\hline T stage & & & 0.136 \\
\hline $\mathrm{T} 2$ & $48(19.4)$ & $20(12.6)$ & \\
\hline T3 & $45(18.2)$ & $37(23.3)$ & \\
\hline T4 & $154(62.3)$ & $102(64.2)$ & \\
\hline N stage & & & 0.593 \\
\hline NO & $60(24.3)$ & $38(23.9)$ & \\
\hline N1 & $51(20.6)$ & $25(15.7)$ & \\
\hline N2 & $43(17.4)$ & $28(17.6)$ & \\
\hline N3 & $93(37.7)$ & $68(42.8)$ & \\
\hline TNM stage & & & 0.724 \\
\hline $\mathrm{lb}$ & $17(6.9)$ & $8(5.0)$ & \\
\hline \|la & $24(9.7)$ & $16(10.1)$ & \\
\hline$\| \mathrm{b}$ & $45(18.2)$ & $30(18.9)$ & \\
\hline IIla & $47(19.0)$ & $22(13.8)$ & \\
\hline$\| l \mid b$ & $42(17.0)$ & $30(18.9)$ & \\
\hline IIIC & $72(29.1)$ & $53(33.3)$ & \\
\hline \multicolumn{4}{|l|}{ No.4D LNs } \\
\hline Number of positive & $0.7 \pm 1.3$ & $0.8 \pm 1.8$ & 0.675 \\
\hline Number of examined & $3.7 \pm 2.7$ & $4.9 \pm 4.0$ & 0.001 \\
\hline \multicolumn{4}{|l|}{ No.4SB LNs } \\
\hline Number of positive & $0.1 \pm 0.5$ & $0.1 \pm 0.8$ & 0.617 \\
\hline Number of examined & $1.7 \pm 2.2$ & $1.6 \pm 2.3$ & 0.743 \\
\hline \multicolumn{4}{|l|}{ No.6 LNs } \\
\hline Number of positive & $1.2 \pm 2.0$ & $0.9 \pm 1.7$ & 0.158 \\
\hline Number of examined & $4.4 \pm 3.4$ & $4.8 \pm 3.8$ & 0.362 \\
\hline \multicolumn{4}{|l|}{ Total } \\
\hline Number of positive & $5.9 \pm 6.4$ & $7.5 \pm 8.7$ & 0.045 \\
\hline Number of examined & $25.4 \pm 9.9$ & $40.6 \pm 17.5$ & $<0.001$ \\
\hline
\end{tabular}

LNs lymph nodes

was $11.4 \pm 4.4$ days in bursectomy group and $11.4 \pm 4.4$ days in non-bursectomy group $(p=0.850)$. Postoperative morbidity rate was comparable between the bursectomy and the non-bursectomy groups (26.4 vs. $17.8 \%, p=0.179)$. Details of postoperative complications were listed in Table 3. Reoperation was needed in five patients within 30 days of operation: three patients for abdominal cavity hemorrhage and two patients for intra-abdominal abscesses in bursectomy group and two patients for abdominal cavity hemorrhage and another patient of intestinal obstruction in non-bursectomy group. There was just one hospital death; a patient in non-bursectomy group died of postoperative acute myocardial infarction. All patients recovered well and successfully discharged from the hospital, 
Table 3 Short-term outcomes of the non-bursectomy group and the bursectomy group

\begin{tabular}{|c|c|c|c|}
\hline & $\begin{array}{l}\text { Non-bursectomy } \\
\text { group }\end{array}$ & $\begin{array}{l}\text { Bursectomy } \\
\text { group }\end{array}$ & $p$ value \\
\hline & $N=247(\%)$ & $N=159(\%)$ & \\
\hline $\begin{array}{l}\text { Postoperative hospital } \\
\text { stay (days) }\end{array}$ & $11.4 \pm 4.4$ & $11.4 \pm 4.4$ & 0.850 \\
\hline Overall morbidity & $44(17.8)$ & $37(23.3)$ & 0.179 \\
\hline PPCs & $19(43.18)$ & $15(40.5)$ & \\
\hline Pancreatic fistula & $4(9.1)$ & $5(13.5)$ & \\
\hline Gastroparesis & $5(11.4)$ & $3(8.1)$ & \\
\hline $\begin{array}{l}\text { Intraperitoneal } \\
\text { hemorrhage }\end{array}$ & $3(6.8)$ & $5(13.5)$ & \\
\hline Anastomotic leakage & $1(2.3)$ & $1(2.7)$ & \\
\hline SSIs & $3(6.8)$ & $1(2.7)$ & \\
\hline Intraperitoneal infection & $5(11.4)$ & $6(16.2)$ & \\
\hline Postoperative ileus & $3(6.8)$ & $1(2.7)$ & \\
\hline $\begin{array}{l}\text { Acute myocardial } \\
\text { infarction }\end{array}$ & $1(2.3)$ & $0(0)$ & \\
\hline \multicolumn{4}{|l|}{ Postoperative mortality } \\
\hline \multicolumn{2}{|c|}{ Classification of complications $^{\mathrm{a}}$} & & 0.759 \\
\hline Grade I & $21(47.7)$ & $16(43.2)$ & \\
\hline Grade II & $19(43.2)$ & $16(43.2)$ & \\
\hline Grade III & $2(4.5)$ & $3(8.1)$ & \\
\hline Grade IV & $1(2.3)$ & $2(5.4)$ & \\
\hline Grade V & $1(2.3)$ & $0(0)$ & \\
\hline
\end{tabular}

Abbreviations: PPCS postoperative pulmonary complications, SS/s, surgical site infections

${ }^{\mathrm{a}}$ The Clavien-Dindo classification of surgical complications

including one patient in bursectomy group and one patient in non-bursectomy group who had a duodenal stump fistula with full drainage and effective nutrition support with more than 30-day postoperative hospital stay. We also compared the grades of postoperative complications according to the Clavien-Dindo classification and found that it was comparable between the two groups, $p=0.783$ [19].

\section{Survival outcomes}

For the postoperative follow-up, there were 382 patients with fully postoperative follow-up information and a $94.1 \%$ follow-up rate, 20 (2-35) months median follow-up duration. Survival benefits can be observed in bursectomy group compared to non-bursectomy group, although without significant difference, $p=0.055$ (Fig. 3). In the univariate survival analysis, the longitudinal location $(p=0.030)$, macroscopic type $(p=0.027)$, tumor size $(p=0.002)$, resection patterns $(p=0.012)$, $\mathrm{pT}$ stage $(p<0.001)$, and $\mathrm{pN}$ stage $(p<0.001)$ were prognostic risk factors for the overall survival. And in the multivariate analysis, bursectomy (without vs. with, $p<0.001$ ), $\mathrm{pT}$ stage (pT2-3 stages vs. pT4 stage, $p<0.001$ ), and $\mathrm{pN}$ stage (N0 vs. N3, $p=0.002$ ) were independent prognostic risk factors for the overall survival (Table 4). Subgroup analyses were conducted in the resection patterns and $\mathrm{pT}$ stages. For those patients who underwent distal gastrectomy, the survival outcome was comparable between the two groups $(p=0.129)$, whereas the bursectomy group had better prognosis of patients who underwent total gastrectomy than patients in nonbursectomy group ( $p=0.016$ ) (Fig. 4a, b). There is no significant difference between the two groups for patients with pT2-3 stages $(p=0.117)$ and pT4 stages $(p=0.128)$ (Fig. 5a, b). And for patients with pT4 stage, although there exist differences in the survival curves, no significant differences are shown for patients who underwent distal gastrectomy $(p=0.154)$ and total gastrectomy $(p=0.160)($ Fig. $6 \mathrm{a}, \mathrm{b})$

\section{Discussion}

In the 1960s, bursectomy is seen as an essential component of radical surgery for serosa-involved gastric adenocarcinomas in Japan. However, surgical safety and oncologic benefits are necessary factors in order to make sure bursectomy as a potential useful therapeutic procedure in the gastric cancer surgery according to the viewpoints today. By the results of the previous study, safety of bursectomy with D2 lymphadenectomy strongly hinges on the experience of surgeons [20]. With regard to the long-term survival outcomes, there was only one RCT that suggested that bursectomy had some survival benefits among the serosa-positive (pT3-T4) patients and without significant difference, the 3-year overall survival rate was $69.8 \%$ for the bursectomy patients, in contrast to $50.2 \%$ for the nonbursectomy group [9], and the 5-years follow-up results of this study existed similar results [21]. On the other hand, other studies had totally opposite results, and they did not find survival benefits of bursectomy when compared with non-bursectomy surgery [10-12]. Moreover, in China, more than half of gastric cancer patients were with advanced stage tumors. Therefore, we assessed the outcomes of advanced gastric cancer patients who underwent D2 gastrectomy with bursectomy or who are not in a single institute of China. In this study, we found that the postoperative complications rate was comparable between patients with or without bursectomy, and D2 gastrectomy with bursectomy had benefits in short-term overall survival outcomes when compared with non-bursectomy gastrectomy, especially for patients who underwent total gastrectomy.

Bursectomy is a complicated and technique-dependent procedure, which may increase the surgical duration and account for more blood loss during the operations. The previous Japanese randomized controlled trial found that bursectomy was associated with an additional $27 \mathrm{~min}$ surgical duration and an additional $125 \mathrm{ml}$ intraoperative blood loss compared with non-bursectomy [20]. And another cohort study found that bursectomy procedure was associated with an additional 41 min operation 


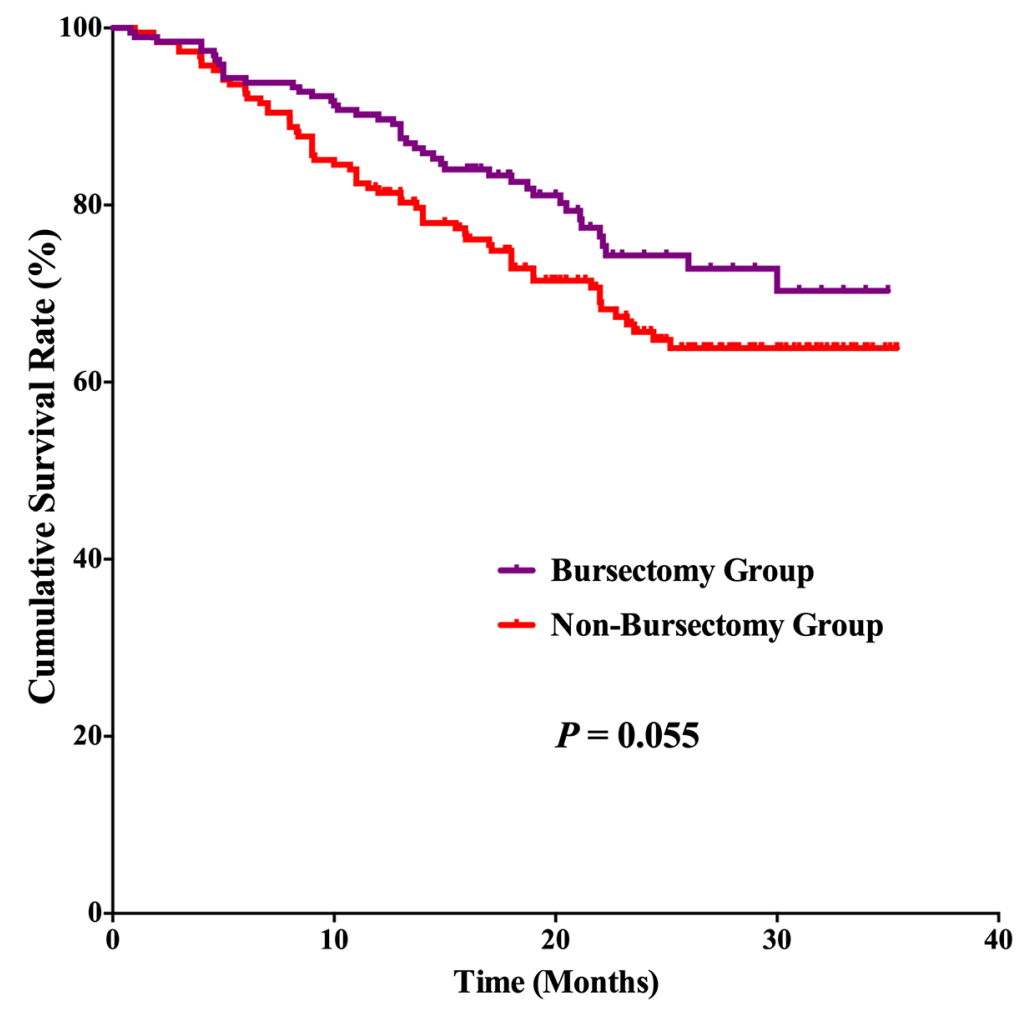

Fig. 3 Survival curves among the bursectomy group and non-bursectomy group. There was no significant difference in survival between the two groups $(p=0.055)$

times and an additional $65 \mathrm{ml}$ intraoperative blood loss [22]. Also in our study, increasing of the surgical duration can be discovered in bursectomy group than the non-bursectomy group. The extra time consuming of the operations was mainly because of the dissection of the anterior of mesocolon of transverse and capsule of the pancreas. However, long surgical duration and high intraoperative blood loss do not mean the unsafety of the surgical bursectomy procedures although with the potential injury of the vessels of the mesocolon of transverse. Blouhos et al. [22] found that the postoperative morbidity rate was $19.4 \%$ for patients with bursectomy, and Imamura et al. [20], in their study, presented that the overall morbidity rate was $14.3 \%$ for both the bursectomy and non-bursectomy. In our study, the incidence of postoperative complications was comparable with the two groups (bursectomy group $23.3 \%$ vs. non-bursectomy group $17.8 \%, p=0.179$ ). Meanwhile, the grade of complications according to the ClavienDindo surgical complications classification [19] between the two groups was also comparable $(p=0.759)$. Therefore, despite the fact that the bursectomy is a time-consuming procedure, the D2 lymphadenectomy with gastrectomy plus bursectomy can be performed safely in high-volume experienced centers or by experienced surgeons [9].
Of the postoperative complications, in details, gastrointestinal surgeons are most concerned about the possible damage of the pancreas and potential trend increase the incidence of pancreatic fistula formation and postoperative intestinal obstruction. Injury to the pancreatic parenchyma may occur when dissecting the pancreatic capsule and lead to the probable incidence of pancreatic fistula. Previous study reported that subclinical pancreatic fistula could occur in up to $10 \%$ of the patients with the resection of the pancreatic capsule [23]. And on the other hand, Imamura et al., in their study, observed that there was no difference in the incidence of pancreatic fistulas and amylase levels in the postoperative drainage fluid between the bursectomy group and non-bursectomy group [20]. They concluded that pancreatic fistula may not be caused by the dissection of the pancreatic capsule but owing to the lymphadenectomy of those lymph nodes adjacent to the pancreas parenchyma. Blouhos et al. reported that the incidence rate of pancreatic fistula is only $4.2 \%(3 / 72)$ [22]. Generally, the resection of the pancreatic capsule is experienced cumulative procedures, and the experienced surgeons in an experienced center can rarely induce injury to the pancreas and reduce the potential incidence of pancreatic fistula [22].

Next, concerned postoperative adverse events are the possibility of intra-abdominal adhesion formation and the potential intra-abdominal intestinal adhesive obstruction. 
Table 4 The univariate and multivariate survival analysis of all patients

\begin{tabular}{|c|c|c|c|}
\hline & $p$ value ${ }^{*}$ & $\begin{array}{l}\text { Multivariate } \\
\text { HR (95 \% Cl) }\end{array}$ & $p$ value \\
\hline$\overline{\text { Age }}$ & 0.753 & & \\
\hline \multicolumn{4}{|l|}{$<65$ years vs. $\geq 65$ years } \\
\hline Gender & 0.880 & & \\
\hline \multicolumn{4}{|l|}{ Male vs. female } \\
\hline Cross-sectional location & 0.315 & & \\
\hline \multicolumn{4}{|l|}{$\begin{array}{l}\text { Lesser vs. greater vs. anterior } \\
\text { vs. posterior vs. circumferential } \\
\text { involvement }\end{array}$} \\
\hline Longitudinal location & 0.030 & & \\
\hline \multicolumn{4}{|l|}{ U vs. M vs. L } \\
\hline Differentiation grade & 0.378 & & \\
\hline \multicolumn{4}{|l|}{$\begin{array}{l}\text { Well-moderate vs. } \\
\text { poor-undifferentiated }\end{array}$} \\
\hline Macroscopic type & 0.027 & & \\
\hline \multicolumn{4}{|l|}{ Type 1-2 vs. Type 3-4 } \\
\hline Tumor size & 0.002 & & \\
\hline \multicolumn{4}{|l|}{$<5 \mathrm{~cm}$ vs. $\geq 5 \mathrm{~cm}$} \\
\hline \multicolumn{4}{|l|}{ Resection patterns } \\
\hline DG vs. TG & 0.012 & & \\
\hline Bursectomy & 0.055 & & 0.025 \\
\hline With vs. without & & $\begin{array}{l}1: 1.640 \\
(1.064-2.528)\end{array}$ & \\
\hline T stage & $<0.001$ & 1 & \\
\hline pT2-3 vs. pT4 & & $\begin{array}{l}1: 2.719 \\
(1.615-4.579)\end{array}$ & $<0.001$ \\
\hline N stage & $<0.001$ & & \\
\hline No & & 1 & \\
\hline $\mathrm{N} 1$ & & $\begin{array}{l}1.674 \\
(0.812-3.450)\end{array}$ & 0.163 \\
\hline N2 & & $\begin{array}{l}1.936 \\
(0.934-4.010)\end{array}$ & 0.075 \\
\hline N3 & & $\begin{array}{l}2.702 \\
(1.461-4.997)\end{array}$ & 0.002 \\
\hline
\end{tabular}

Abbreviations: $U$ upper, $M$ middle, $L$ lower, $D G$ distal gastrectomy, $T G$ total gastrectomy

*Log-rank test; ${ }^{* *}$ Cox hazard model

For the gastrectomy with bursectomy, adhesions to the mesocolon and pancreas may cause specific symptoms, such as delayed gastric emptying, afferent loop syndrome, or intestinal obstruction [20]. The early stage postoperative intestinal obstruction usually occurs in about 1-2 weeks after the operation. However, postoperative intestinal obstruction can occur at any point in time during the postoperative period. Therefore, short-term postoperative observation is not enough to fully evaluate the incidence of postoperative intestinal obstruction. Long-term follow-up and careful observation in a large sample size cohort study is expected to draw the conclusion about postoperative intestinal obstruction of bursectomy.

The most important purpose of total bursectomy is to eliminate the cancer cells for the prevention of potential peritoneal relapse and improve the survival outcomes. However, evidence of survival outcomes decides whether "bigger surgery is better." For the tumor that penetrates the serosa of the stomach, some surgeons hold the attitude that bursectomy cannot eliminate all disseminated free cancer cells because the bursa omentalis is not a closed space and bursectomy is unlikely to improve overall survival in patients with subserosa or serosa invasive cancers [10]. Previous studies found that there was no statistical difference between the two groups in overall survival, and the bursectomy procedure was not recommended as a routinely procedure [5, 10-12, 24]. However, we also noticed that the interim analyses and the final reports of Osaka trial found that bursectomy surgery had some survival advantages in stage pT3-4 patients when compared with nonbursectomy surgery regarding the recurrence rate, recurrence type (peritoneal seeding recurrence) and the survival rates $[9,21]$. Meanwhile, we noticed that there is no statistical difference between the recurrence rate and recurrence type between the two groups (Additional file 1: Table S1). Recurrence type is another important factor to evaluate the survival benefits for different resection strategies. These results maybe due to the short-term follow-up duration, which may not completely present the potential recurrence between two groups. There is no difference of survival outcomes between the two groups, but the bursectomy group had better curves than the non-bursectomy group (Fig. 1). The following may be the reasons for these results: the bursectomy may have some benefits in terms of prolonging the patients' survival outcomes, but because of the sample size and limited follow-up duration result, the statistical difference did not appear. In view of these results, besides Japan and Korea, advanced stage gastric cancer account for about half of total gastric cancer patients [25-27], especially in China [28]. Therefore, a well-designed large sample RCTs with long-term study to find out the details operation indications of the bursectomy for advanced gastric cancer patients are expected.

Interestingly, we noticed that the number of harvested lymph nodes was significantly higher in the bursectomy group than the non-bursectomy group (40.6 \pm 17.5 vs. $25.4 \pm 9.9$, respectively, $p<0.001)$. Meanwhile, the number of positive lymph nodes was also higher in the bursectomy group than the non-bursectomy group $(7.5 \pm 8.7$ vs. $5.9 \pm$ 6.4 , respectively, $p=0.045)$. Lymph node dissection strategy is one of the hottest topics regarding gastric cancer surgery. On one hand, because of the limitation of the retrospective study, the potential selection bias may be one reason, which results to the different lymph node results between the two groups. Although, we only include those 

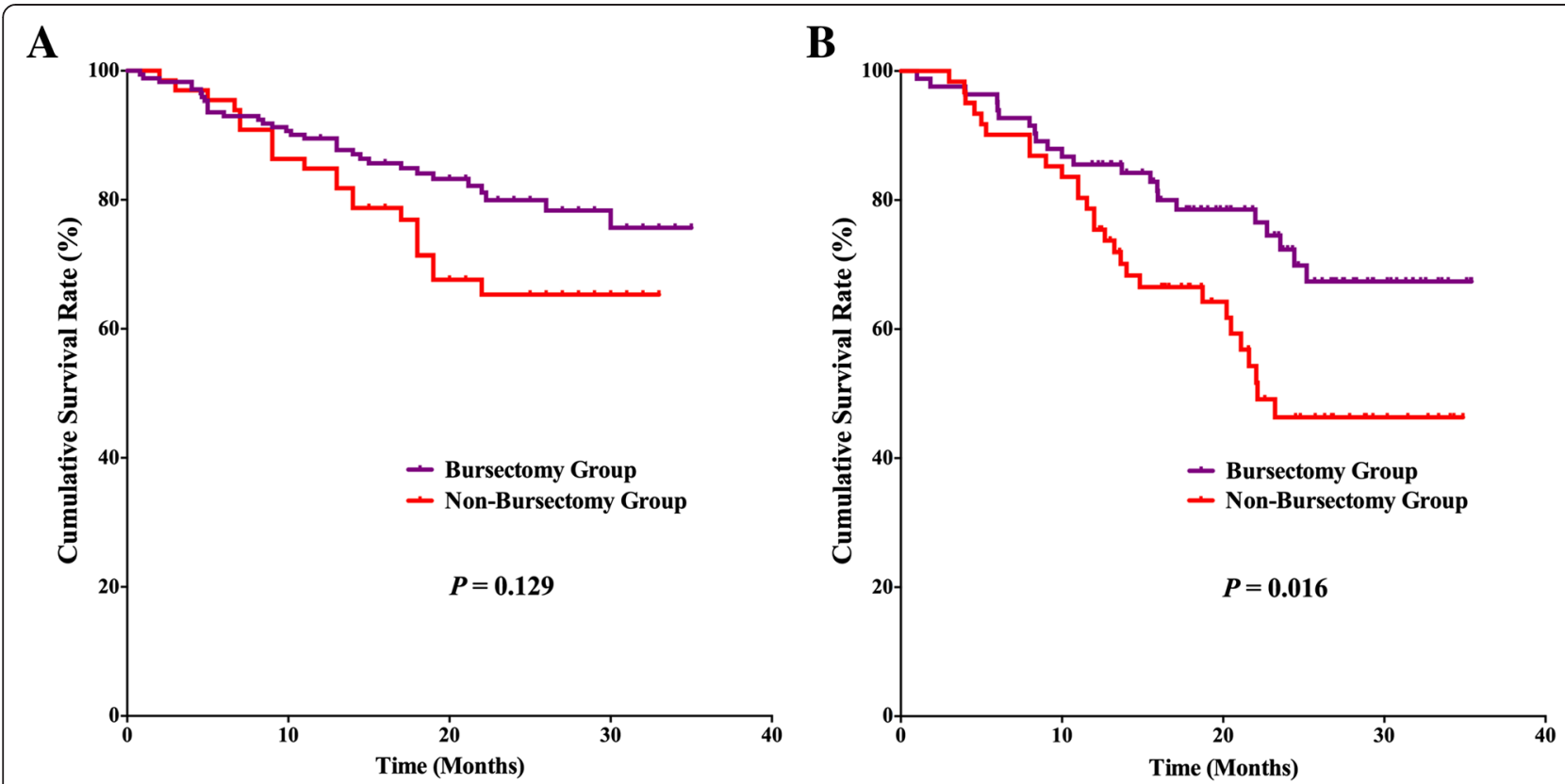

Fig. 4 Survival curves among the bursectomy group and non-bursectomy group for patients who underwent distal gastrectomy (a) and total gastrectomy (b)

patients who underwent surgical treatment by surgeons of gastric cancer surgical treatment team in our department, which aims to dimension the potential bias coming from operators. On the other hand, Greece scholar Blouhos et al. presented right side bursectomy as an access plane for completely en bloc dissection of lymph nodes [29]. This may be another reason for the different lymph node result between the two groups. It is certain that both the Western and Eastern guidelines recommended at least 16 lymph nodes examined for gastric cancer surgery in the $\mathrm{N}$ stage determination [30]. In our study, the non-bursectomy dissected $25.4 \pm 9.9$ lymph nodes, which is higher than the recommendation of the guidelines. Also, some study reported that the number of lymph node dissection is closely
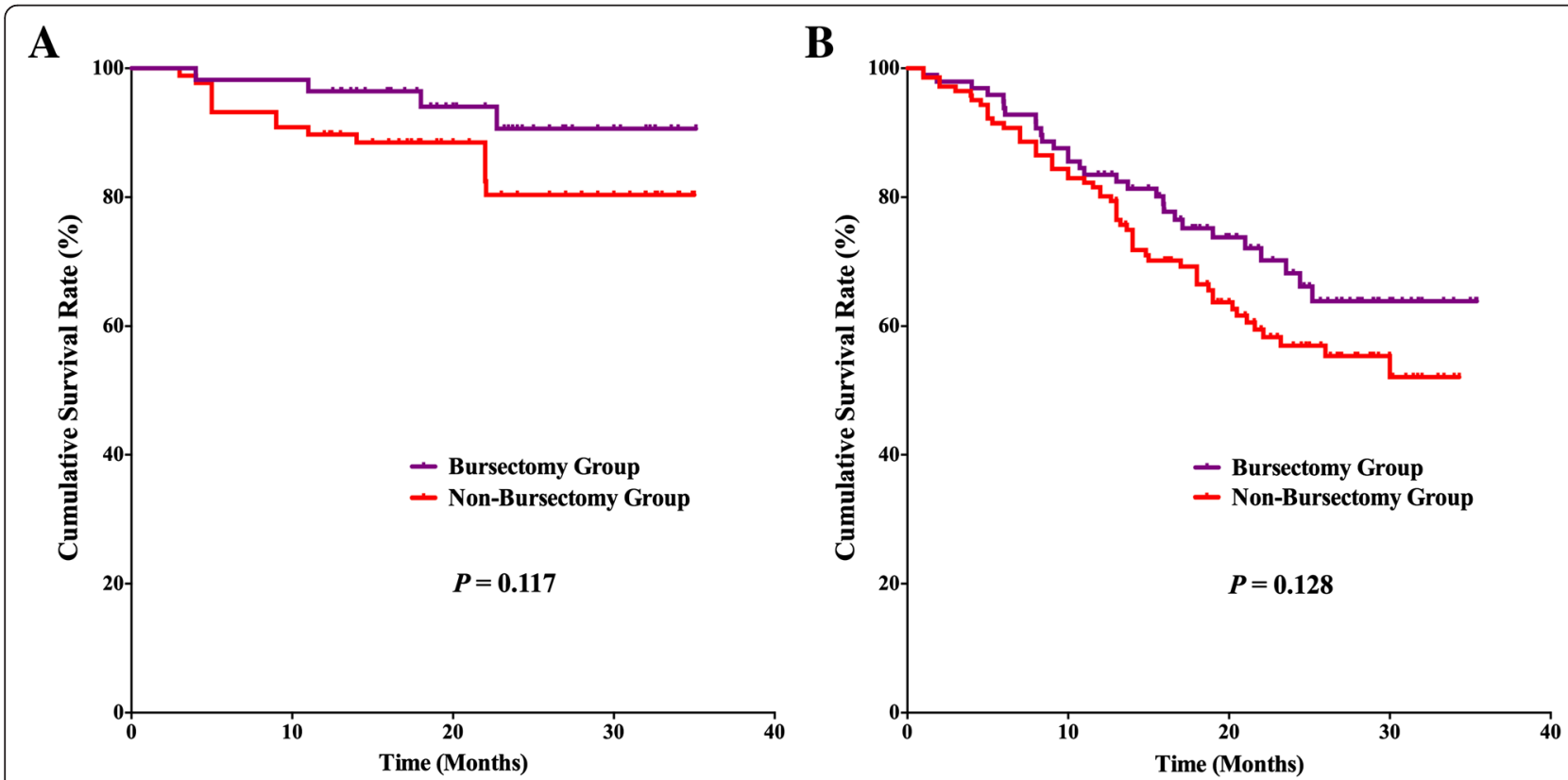

Fig. 5 Survival curves among the bursectomy group and non-bursectomy group for patients with pT2-3 (a) stages and pT4a stages (b) 

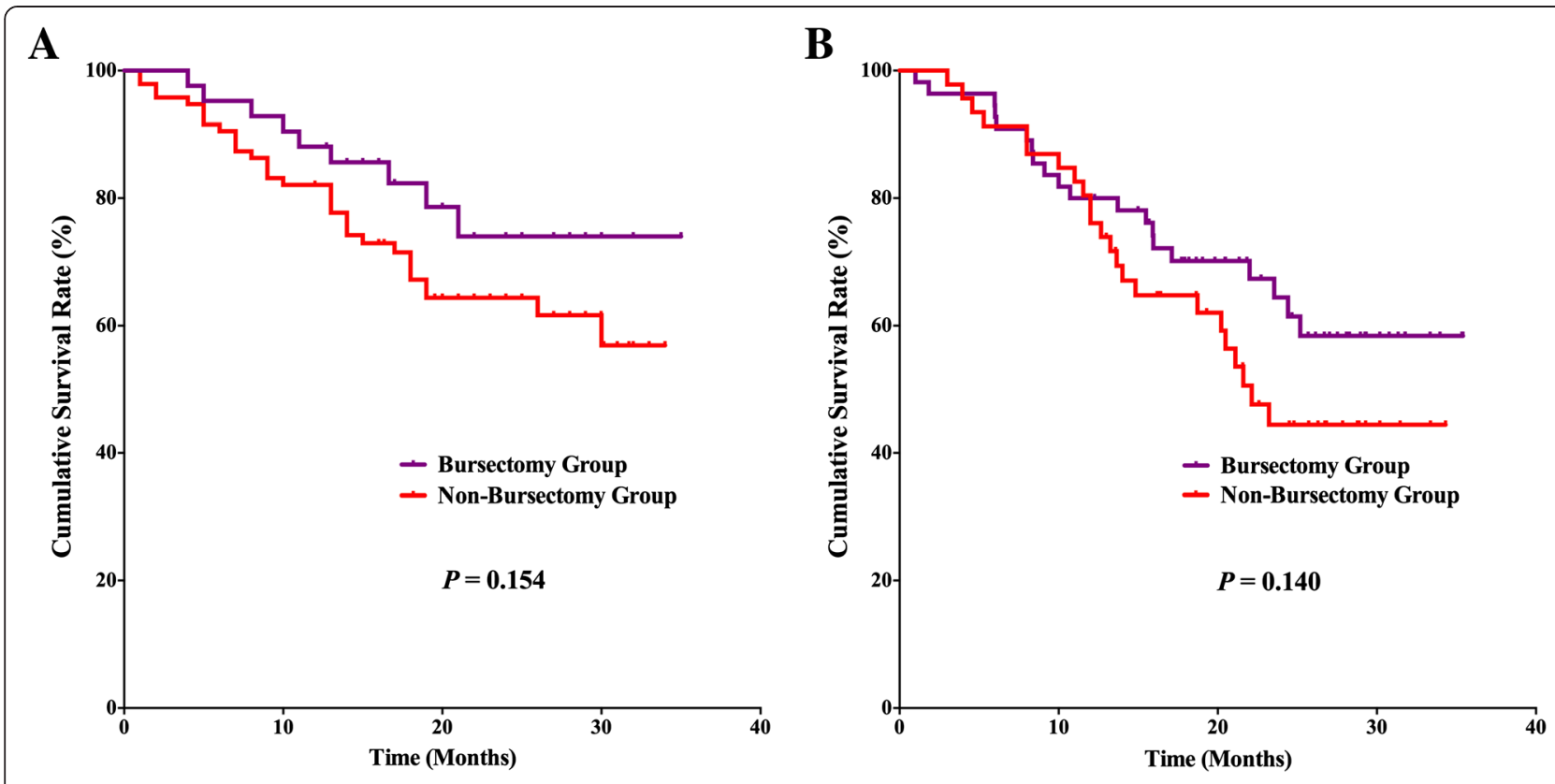

Fig. 6 Survival curves among the bursectomy group and non-bursectomy group for patients with pT4a stage who underwent distal gastrectomy (a) and total gastrectomy (b)

related with the prognosis of patients [31-34]. Previous study identified that for stage N2-N3 gastric cancer patients, harvesting at least 25 lymph nodes may represent a superior cutoff for radical gastrectomy and could yield better survival outcomes [35]. Therefore, regardless of the fact that there existed a significant difference in the number of lymph nodes between the two groups, the number of examined lymph nodes in non-bursectomy is higher than the requirements of the present gastric cancer classification.

Meanwhile, different from the previous study, we included those patients who underwent total gastrectomy. Although, the results of this study found that there was no significant difference in the short-term survival outcomes between bursectomy and non-bursectomy groups. For those patients who underwent total gastrectomy, the survival benefit of the bursectomy has appeared. Note that despite that there is no significant difference in survival outcomes for distal gastrectomy patients or pT4 stage patients, the survival curves had demonstrated the separated trending between the two surgical strategies. As the previous description, sample size and short-term follow-up duration may be the reasons for these survival results. Also, upper third gastric cancers usually had poor tumor characteristics and survival outcomes than lower third gastric cancers [36]. Besides, those lower third gastric cancers that underwent total gastrectomy are generally due to the poor tumor characteristics. These may be the main reasons why there exists a statistical difference between the two groups in total gastrectomy and not in distal gastrectomy. Despite the fact that this study set ups subgroup analysis in the resection patterns and conducts the multivariate survival analysis which identified that bursectomy or not is one of the independent prognostic risk factors, the potential selection bias of the retrospective study was existing and which must be emphasized here again. Regardless of the fact that previous and present evidence did not strongly prove that the bursectomy should be routinely performed, this technique-dependent procedure may be existing survival benefits in gastric cancer patients with specific characteristics, such as $\mathrm{T}$ stages, tumor locations, or other characteristics. Thus, well-designed RCTs are expected to clear these.

\section{Conclusions}

As a highly technique-dependent procedure, bursectomy resulted in longer surgical duration than non-bursectomy surgery. Experienced surgeons could safely perform D2 gastrectomy with bursectomy and the postoperative complications not increased. Whether the bursectomy has survival benefits for all or special characteristics gastric cancer patient and surgical indications of bursectomy surgery is pending.

\section{Additional file}

Additional file 1: Table S1. Tumor recurrence between the two groups.

\section{Abbreviations}

LNs: lymph nodes; RCT: randomized controlled trial; JGCA: Japanese Gastric Cancer Association; UICC: Union for International Cancer Control;

TNM: tumor-node-metastasis; JCOG: Japan Clinical Oncology Group. 


\section{Competing interests}

The authors declare that they have no competing interests.

\section{Authors' contributions}

$\mathrm{W}-\mathrm{HZ}$ performed the analyses and wrote the article. $\mathrm{X}-\mathrm{ZC}$ collected the operation photos. $\mathrm{W}-\mathrm{HZ}, \mathrm{KY}$, and $\mathrm{KL}$, collected the data; $\mathrm{Z}-\mathrm{XC}$ and $\mathrm{BZ}$ performed the operations. Z-GZ proofread the article. J-KH performed the operations and reviewed the article. All authors read and approved the final manuscript.

\section{Authors' information}

The corresponding author Jian-Kun Hu, M.D., Ph.D., FRCS, is the vice director of Gastrointestinal Surgery department, West China Hospital, Sichuan University, Director of Laboratory of Gastric Cancer, State Key Laboratory of Biotherapy, West China Hospital, Sichuan University. His expertise includes surgical treatment of gastric cancer and the Research on Stem Cell of Gastrointestinal Epithelium.

\section{Acknowledgements}

The authors thank the Volunteer Team of Gastric Cancer Surgery based on the Multidisciplinary Team of Gastrointestinal Tumors, West China Hospital, Sichuan University, China, for their substantial work.

\section{Funding}

Domestic support from the (1) National Natural Science Foundation of China (No. 81372344); (2) New Century Excellent Talents in University support program, Ministry of Education of China (2012SCU-NCET-11-0343).

\section{Received: 7 June 2015 Accepted: 27 November 2015}

\section{Published online: 08 December 2015}

\section{References}

1. Torre LA, Bray F, Siegel RL, Ferlay J, Lortet-Tieulent J, Jemal A. Global cancer statistics, 2012. CA Cancer J Clin. 2015;65(2):87-108. doi:10.3322/caac.21262. PubMed.

2. Colquhoun A, Arnold M, Ferlay J, Goodman KJ, Forman D, Soerjomataram I. Global patterns of cardia and non-cardia gastric cancer incidence in 2012. Gut. 2015. doi:10.1136/gutjnl-2014-308915.PubMed.

3. Chen W, Zheng R, Zeng H, Zhang S, He J. Annual report on status of cancer in China, 2011. Chin J Cancer Res. 2015;27(1):2-12. Epub 2015/02/27. doi: 10. 3978/j.issn.1000-9604.2015.01.06. PubMed PMID: 25717220; PubMed Central PMCID: PMC4329176

4. Groves EW. On the radical operation for cancer of the pylorus: with especial reference to the advantages of the two-stage operation and to the question of the removal of the associated lymphatics. Br Med J. 1910; 1(2563):366-70. PubMed PMID: 20764919, PubMed Central PMCID: PMC2330344, Epub 1910/02/12.

5. Yoshikawa T, Tsuburaya A, Kobayashi O, Sairenji M, Motohashi H, Hasegawa $S$, et al. Is bursectomy necessary for patients with gastric cancer invading the serosa? Hepato-Gastroenterology. 2004;51(59):1524-6. Epub 2004/09/15. PubMed.

6. Hundahl SA. The potential value of bursectomy in operations for trans-serosal gastric adenocarcinoma. Gastric Cancer. 2012;15(1):3-4. doi:10.1007/s10120011-0121-6. Epub 2011/12/14. PubMed.

7. Blouhos K, Boulas KA, Tsalis K, Hatzigeorgiadis A. Right-sided bursectomy as an access plane for aesthetic resection of the posterior leaf of the lesser sac from the head of the pancreas en block with the No. 6 and 14v lymph nodes in advanced lower third gastric cancer. Surgery. 2015. Epub 2015/02/24. doi: 10.1016/j.surg.2014.12.008. PubMed

8. Fukuda N, Sugiyama Y, Wada J. Prognostic factors of T4 gastric cancer patients undergoing potentially curative resection. World J Gastroenterol. 2011;17(9): 1180-4. doi:10.3748/wjg.v17.i9.1 180. PubMed PMID: 21448423, PubMed Central PMCID: PMC3063911.

9. Fujita J, Kurokawa Y, Sugimoto T, Miyashiro I, lijima S, Kimura Y, et al. Survival benefit of bursectomy in patients with resectable gastric cancer: interim analysis results of a randomized controlled trial. Gastric Cancer. 2012; 15(1):42-8. doi:10.1007/s10120-011-0058-9. Epub 2011/05/17. PubMed.

10. Eom BW, Joo J, Kim YW, Bae JM, Park KB, Lee JH, et al. Role of bursectomy for advanced gastric cancer: result of a case-control study from a large volume hospital. Eur J Surg Oncol. 2013;39(12):1407-14. doi:10.1016/j.ejso.2013.09.013. Epub 2013/10/15. PubMed.
11. Kochi M, Fujii M, Kanamori N, Kaiga T, Mihara Y, Funada T, et al. D2 gastrectomy with versus without bursectomy for gastric cancer. Am J Clin Oncol. 2014;37(3): 222-6. doi:10.1097/COC.0b013e31825eb734. Epub 2012/08/16. PubMed.

12. Shen WS, Xi HQ, Wei B, Chen L. Effect of gastrectomy with bursectomy on prognosis of gastric cancer: a meta-analysis. World J Gastroenterol. 2014; 20(40):14986-91. doi:10.3748/wjg.v20.i40.14986. PubMed PMID: 25356060, PubMed Central PMCID: PMC4209563, Epub 2014/10/31.

13. Japanese gastric cancer treatment guidelines 2010 (ver. 3). Gastric Cancer. 2011;14(2):113-23. Epub 2011/05/17. doi: 10.1007/s10120-011-0042-4. PubMed

14. Japanese Gastric Cancer A. Japanese classification of gastric carcinoma-2nd English edition. Gastric Cancer. 1998;1(1):10-24. doi:10. 1007/s101209800016. PubMed.

15. Japanese classification of gastric carcinoma: 3rd English edition. Gastric Cancer. 2011;14(2):101-12. Epub 2011/05/17. doi: 10.1007/s10120-011-0041-5. PubMed

16. Japanese Gastric Cancer A. Japanese gastric cancer treatment guidelines 2010 (ver. 3). Gastric Cancer. 2011;14(2):113-23. doi: 10.1007/s10120-011-0042-4. PubMed

17. Washington K. 7th edition of the AJCC cancer staging manual: stomach. Ann Surg Oncol. 2010;17(12):3077-9. doi:10.1245/s10434-010-1362-z. PubMed.

18. Bassi C, Dervenis C, Butturini G, Fingerhut A, Yeo C, Izbicki J, et al. Postoperative pancreatic fistula: an international study group (ISGPF) definition. Surgery. 2005;138(1):8-13. doi:10.1016/j.surg.2005.05.001. Epub 2005/07/09. PubMed.

19. Clavien PA, Barkun J, de Oliveira ML, Vauthey JN, Dindo D, Schulick RD, et al. The Clavien-Dindo classification of surgical complications: five-year experience. Ann Surg. 2009;250(2):187-96. doi:10.1097/SLA.0b013e3181b13ca2. PubMed.

20. Imamura H, Kurokawa Y, Kawada J, Tsujinaka T, Takiguchi S, Fujiwara Y, et al. Influence of bursectomy on operative morbidity and mortality after radical gastrectomy for gastric cancer: results of a randomized controlled trial. World J Surg. 2011;35(3):625-30. doi:10.1007/s00268-010-0914-5. Epub 2010/12/17. PubMed.

21. Hirao M, Kurokawa Y, Fujita J, Imamura H, Fujiwara Y, Kimura Y, et al. Longterm outcomes after prophylactic bursectomy in patients with resectable gastric cancer: final analysis of a multicenter randomized controlled trial. Surgery. 2015. doi:10.1016/j.surg.2014.12.024. Epub 2015/02/24. PubMed.

22. Blouhos K, Boulas KA, Hatzigeorgiadis A. Metastasis in lymph nodes on the anterior pancreatic surface of the body and tail: an extremely rare finding during bursectomy in extended surgery for gastric cancer. Updates Surg. 2014. doi:10.1007/s13304-014-0276-x. Epub 2014/12/20. PubMed.

23. Herbella FA, Tineli AC, Wilson Jr JL, Del Grande JC. Gastrectomy and lymphadenectomy for gastric cancer: is the pancreas safe? J Gastrointest Surg. 2008;12(11):1912-4. doi:10.1007/s1 1605-008-0572-1. Epub 2008/07/10. PubMed.

24. Yamamura Y, Ito S, Mochizuki Y, Nakanishi H, Tatematsu M, Kodera Y. Distribution of free cancer cells in the abdominal cavity suggests limitations of bursectomy as an essential component of radical surgery for gastric carcinoma. Gastric Cancer. 2007;10(1):24-8. doi:10.1007/s10120-006-0404-5. Epub 2007/03/06. PubMed.

25. Nashimoto A, Akazawa K, Isobe Y, Miyashiro I, Katai H, Kodera Y, et al. Gastric cancer treated in 2002 in Japan: 2009 annual report of the JGCA nationwide registry. Gastric Cancer. 2013;16(1):1-27. doi:10.1007/s10120-0120163-4. PubMed PMID: 22729699, PubMed Central PMCID: PMC3549249.

26. Ferro A, Peleteiro B, Malvezzi M, Bosetti C, Bertuccio P, Levi F, et al. Worldwide trends in gastric cancer mortality (1980-2011), with predictions to 2015, and incidence by subtype. Eur J Cancer. 2014;50(7):1330-44. doi:10.1016/j.ejca.2014.01.029. Epub 2014/03/22. PubMed.

27. Hamashima C, Ogoshi K, Narisawa R, Kishi T, Kato T, Fujita K, et al. Impact of endoscopic screening on mortality reduction from gastric cancer. World J Gastroenterol. 2015;21(8):2460-6. doi:10.3748/wjg.v21.i8.2460. PubMed PMID: 25741155, PubMed Central PMCID: PMC4342924, Epub 2015/03/06.

28. Zhang WH, Chen XZ, Liu K, Chen XL, Yang K, Zhang B, et al. Outcomes of surgical treatment for gastric cancer patients: 11-year experience of a Chinese high-volume hospital. Med Oncol. 2014;31(9):150. doi:10.1007/s12032-014-0150-1. PubMed.

29. Blouhos K, Boulas KA, Tsalis K, Hatzigeorgiadis A. Right-sided bursectomy as an access plane for aesthetic resection of the posterior leaf of the lesser sac from the head of the pancreas en block with the No. 6 and 14v lymph nodes in advanced lower third gastric cancer. Surgery. 2015;158(6):1742. doi:10.1016/j.surg.2014.12.008. PubMed.

30. Japanese Gastric Cancer A. Japanese classification of gastric carcinoma: 3rd English edition. Gastric Cancer. 2011;14(2):101-12. doi:10.1007/s10120-011-0041-5. PubMed. 
31. Smith DD, Schwarz RR, Schwarz RE. Impact of total lymph node count on staging and survival after gastrectomy for gastric cancer: data from a large US-population database. J Clin Oncol. 2005;23(28):7114-24. doi:10.1200/JCO.2005.14.621. PubMed.

32. Giuliani A, Caporale A, Corona M, Di Bari M, Demoro M, Ricciardulli T, et al. Lymphadenectomy in gastric cancer: influence on prognosis of lymph node count. J Exp Clin Cancer Res. 2004;23(2):215-24. PubMed.

33. Shen JY, Kim S, Cheong JH, Kim Yl, Hyung WJ, Choi WH, et al. The impact of total retrieved lymph nodes on staging and survival of patients with pT3 gastric cancer. Cancer. 2007;1 10(4):745-51. doi:10.1002/cncr.22837. PubMed.

34. Chen XZ, Yang K, Zhang B, Hu JK, Zhou C. Is retrieval of $>25$ lymph nodes a superior criterion for locally advanced gastric cancer surgery? Ann Surg. 2011; 254(5):834-5. doi:10.1097/SLA.0b013e318235dfda. author reply 5. PubMed.

35. Chen HN, Chen XZ, Zhang WH, Chen XL, Yang K, Liu JP, et al. Necessity of harvesting at least 25 lymph nodes in patients with stage N2-N3 resectable gastric cancer: a 10-year, single-institution cohort study. Medicine. 2015; 94(10):e620. doi:10.1097/MD.0000000000000620. PubMed.

36. Liu K, Zhang W, Chen X, Chen X, Yang K, Zhang B, et al. Comparison on clinicopathological features and prognosis between esophagogastric junctional adenocarcinoma (Siewert II/III Types) and distal gastric adenocarcinoma: retrospective cohort study, a Single Institution, High Volume Experience in China. Medicine. 2015;94(34):e1386. doi: 10.1097/MD.0000000000001386. PubMed PMID: 26313779; PubMed Central PMCID: PMCPMC4602903.

\section{Submit your next manuscript to BioMed Central} and we will help you at every step:

- We accept pre-submission inquiries

- Our selector tool helps you to find the most relevant journal

- We provide round the clock customer support

- Convenient online submission

- Thorough peer review

- Inclusion in PubMed and all major indexing services

- Maximum visibility for your research

Submit your manuscript at www.biomedcentral.com/submit
( BioMed Central 